\title{
Produção de mudas de maracujazeiro-doce micorrizadas em solo desinfestado e adubado com fósforo
}

\author{
Érika Cristina Teixeira dos Anjos ${ }^{(1)}$, Uided Maaze Tiburcio Cavalcante ${ }^{(1)}, V^{(e n e ́ z i o ~ F e l i p e ~ d o s ~ S a n t o s ~}{ }^{(2)}$ \\ e Leonor Costa Maia ${ }^{(1)}$
}

\begin{abstract}
(1)Universidade Federal de Pernambuco, Dep. de Micologia, Av. Prof. Nelson Chaves, s/no, CEP 50670-420 Recife, PE. E-mail: erikaanjos@yahoo.com.br, umaaze@hotmail.com, leonorcmaia@yahoo.com (2)Empresa Pernambucana de Pesquisa Agropecuária, Av. Gal. San Martin, o 1371, CEP 50630-060 Recife, PE. E-mail: venezio@ipa.br
\end{abstract}

Resumo - O objetivo deste trabalho foi selecionar doses de $\mathrm{P}$ e fungos micorrízicos arbusculares (FMA) eficientes na promoção do crescimento de mudas de maracujazeiro-doce. O experimento foi realizado em casa de vegetação, com delineamento inteiramente casualizado, em fatorial $2 \times 4 \times 4$ : duas condições de solo (desinfestado ou não), quatro tratamentos de inoculação (sem inoculação e com inoculação de Gigaspora albida, Scutellospora heterogama e de FMA nativos da rizosfera de maracujazeiro-doce) e quatro doses de P disponível (Mehlich-1) (8 - solo natural -, 12, 16 e $20 \mathrm{mg} \mathrm{dm}^{-3}$ ), com quatro repetições. Os FMA nativos proporcionaram benefícios no crescimento das mudas a partir de 30 dias após a inoculação, enquanto os demais FMA incrementaram o desenvolvimento do hospedeiro após 45 dias. Modelos quadráticos indicaram valores máximos de altura de mudas em solo desinfestado, com dose de P entre 15,40 e 16,07 $\mathrm{mg} \mathrm{dm}^{-3}$ e em solo não desinfestado, com dose de P entre 14,85 e 15,60 $\mathrm{mg} \mathrm{dm}^{-3}$ nos tratamentos com S. heterogama e G. albida, respectivamente. Maiores densidades de esporos e colonização micorrízica foram observadas nos tratamentos com esses fungos, em comparação com os FMA nativos. Mudas de maracujazeiro-doce podem ser beneficiadas pela micorrização com FMA, reduzindo o tempo de sua produção.

Termos para indexação: Passiflora alata, micorriza, fósforo, maracujá.

\section{Production of mycorrhized sweet passion fruit seedlings in disinfected and phosphorus fertilized soil}

\begin{abstract}
The objective of this work was to identify the required P dose and efficient arbuscular mycorrhizal fungi (AMF) to promote growth of seedlings of sweet passion fruit. An experiment was carried out at the greenhouse, in a randomized design with a factorial $2 \times 4 \times 4$ : two soil conditions (disinfected or not), four treatments of inoculation (inoculated or not with Gigaspora albida, Scutellospora heterogama and native AMF from the rhizosphere of sweet passion fruit plants), four doses of available P (Mehlich-1) (8 - natural soil -, 12, 16 and $20 \mathrm{mg} \mathrm{dm}^{-3}$ ), with four replicates. Native AMF promoted growth of seedlings after 30 days of inoculation, while other fungi stimulated plant development after 45 days. Quadratic models indicated maximum values of plant height in soil, with P values estimated between 15.40 and $16.07 \mathrm{mg} \mathrm{dm}^{-3}$ in disinfected soil and 14.85 and $15.60 \mathrm{mg} \mathrm{dm}^{-3}$ in non disinfected soil, obtained in treatments with S. heterogama and G. albida, respectively. Higher spores density and mycorrhizal colonization were observed in treatments with these fungi, comparing with the native AMF. Growth of sweet passion fruit seedlings can be improved by mycorrhization with selected AMF, with reduction of time spent on seedling production.
\end{abstract}

Index terms: Passiflora alata, mycorrhiza, phosphorus, passion fruit.

\section{Introdução}

Os fungos micorrízicos arbusculares (FMA) colonizam quase todas as plantas, sob condições naturais de campo. Tais fungos, além de promoverem o crescimento do hospedeiro, aumentam a tolerância a estresses bióticos e abióticos (Smith \& Read, 1997). Os benefícios da inoculação micorrízica são conseqüência do aumento da zona de absorção das raízes, mediante o desenvolvimento de hifas externas, que crescem além da zona de depleção, favorecendo o aporte de nutrientes, especialmente do fósforo. Este fato ganha importância em solos tropicais onde, em geral, é baixa a disponibilidade deste elemento para as plantas (Novais \& Smyth, 1999).

A desinfestação do solo, prática comum para eliminar patógenos, também elimina propágulos dos fungos micorrízicos, o que tem sido corrigido com a reposição de FMA eficientes, com resultados comprovados em diversas culturas, entre as quais a da aceroleira (Malpighia emarginata D.C.) (Costa et al., 2001) e 
do maracujazeiro-amarelo (Passiflora edulis Sims f. flavicarpa Deg.) (Cavalcante et al., 2002).

A resposta das plantas à micorrização está ligada à temperatura, umidade, $\mathrm{pH}$ do solo (Smith \& Read, 1997) e, principalmente, à fertilidade. Determinar a dose de $\mathrm{P}$ para maximizar a produção ou resposta das plantas é essencial, considerando que esta é diferenciada para cada espécie de fungo e de planta. Geralmente, os maiores benefícios ocorrem em doses moderadas de $\mathrm{P}$ no solo, pois a aplicação elevada desse elemento aumenta os custos de produção e pode inibir a atuação dos FMA (Saggin-Júnior \& Siqueira, 1996).

Para cada hospedeiro específico devem ser testados vários isolados de FMA, pois muitos desses fungos possuem certa preferência associativa (Sieverding, 1991). A inoculação de FMA para produção de mudas tem sido estudada em fruteiras tropicais, tais como mamoeiro (Carica papaya L.) (Trindade et al., 2000), aceroleira (Costa et al., 2001), abacateiro (Persea americana Mill.) (Silveira et al., 2002) e bananeira (Musa sp.) (Yano-Melo et al., 1999).

Isolados de Gigaspora albida Schenck \& Smith e Scutellospora heterogama (Nicol. \& Gerd.) Walker \& Sanders promoveram melhoria no crescimento do maracujazeiro-amarelo. A desinfestação do solo e sua adubação com até $30 \mathrm{mg} \mathrm{dm}^{-3}$ de $\mathrm{P}$ beneficiaram tanto as mudas quanto o crescimento dos FMA (Cavalcante et al., 2002).

O maracujazeiro-doce (Passiflora alata Curtis), com produção ampliada nos últimos anos, especialmente no Estado de São Paulo, onde a produtividade média situa-se entre 25 e 30 t ha $^{-1}$, é atrativo para comercialização no mercado externo, em decorrência das características e qualidades degustativas dos frutos (Vasconcelos et al., 2001).

O objetivo deste trabalho foi selecionar doses de $\mathrm{P} \mathrm{e}$ fungos micorrízicos arbusculares eficientes na promoção do crescimento de mudas de maracujazeiro-doce.

\section{Material e Métodos}

O experimento foi realizado em casa de vegetação do Dep. de Micologia da UFPE, no período de dezembro de 2002 a fevereiro de 2003 , com médias de $28,8^{\circ} \mathrm{C}$ de temperatura e $59 \%$ de umidade relativa do ar. O solo (Latossolo Amarelo distrófico argissólico) apresentou, após desinfestação e diluição com areia (2:1), as seguintes características: $8 \mathrm{mg} \mathrm{dm}^{-3} \mathrm{de} \mathrm{P} ; 0,40,0,75,0,40$ e $0,03 \mathrm{cmol}_{\mathrm{C}} \mathrm{dm}^{-3} \mathrm{de} \mathrm{Al}, \mathrm{Ca}, \mathrm{Mg}$ e $\mathrm{K}$, respectivamente;
$\mathrm{pH}\left(\mathrm{H}_{2} \mathrm{O}\right)$, 4,8; e classe textural franco-arenosa. Parte do substrato foi desinfestado com brometo de metila (150 $\mathrm{mL} \mathrm{m}^{-3}$ ), 20 dias antes de ser utilizado.

Consideraram-se quatro doses de P no solo: 8 (solo natural), 12, 16 e $20 \mathrm{mg} \mathrm{dm}^{-3}$, obtidas com a adição de superfosfato simples, incorporado ao solo antes do plantio. As doses foram escolhidas com base no recomendado (16 $\mathrm{mg} \mathrm{dm}^{-3}$ ) para adubação pela análise de fertilidade do solo.

Para minimizar os efeitos da deficiência mineral do substrato, durante os primeiros 30 dias do experimento as plantas receberam, semanalmente, uma aplicação de solução nutritiva de Hoagland \& Arnon modificada (Jarstfer \& Sylvia, 1992), isenta de fósforo.

Na inoculação, foram utilizados FMA introduzidos (G. albida - UFPE 01 - e S. heterogama - UFPE 12), que foram multiplicados em vasos de cultura, tendo como hospedeiro o painço (Panicum miliacium L.), além de FMA nativos, obtidos da rizosfera de maracujazeiro-doce em plantio comercial, localizado em Limoeiro, PE.

$\mathrm{Na}$ identificação das espécies de FMA nativos, esporos extraídos do solo rizosférico foram agrupados pela semelhança morfológica e montados em lâminas com PVLG (álcool polivinílico). A identificação foi baseada em Schenck \& Perez (1990) e em descrições segundo a International Culture Collection of Vesicular Arbuscular Mycorrhizal Fungi (2003).

As sementes foram desinfestadas com hipoclorito de sódio $20 \%$ por dois minutos e germinadas em bandejas com solo desinfestado. Após o surgimento de duas folhas definitivas, as mudas, exceto as do controle, receberam solo inóculo na região das raízes, correspondendo a 200 esporos por planta de G. albida, S. heterogama ou do inóculo misto com FMA nativos. Quinze dias após a inoculação, as plântulas foram transferidas para sacos de polietileno preto contendo $1.700 \mathrm{~g}$ de substrato.

O delineamento experimental foi inteiramente casualizado em esquema fatorial de $2 \times 4 \times 4$, correspondendo a duas condições de solo (desinfestado ou não), quatro tratamentos de inoculação (sem inoculação e com inoculação de G. albida, $S$. heterogama e FMA nativos), quatro doses de $\mathrm{P}$ $\left(8,12,16\right.$ e $\left.20 \mathrm{mg} \mathrm{dm}^{-3}\right)$ e quatro repetições.

Quinzenalmente foram avaliados a altura, o diâmetro do caule e o número de folhas e, após 65 dias, além destes, foram avalidadas a biomassa após secagem em estufa a $60^{\circ} \mathrm{C}$, área foliar, utilizando-se o Programa Sigma Scan Pro 5 (Jorge et al., 1996), colonização micorrízica e densidade de esporos na rizosfera. A colonização 
micorrízica foi estimada em 100 fragmentos de $1 \mathrm{~cm}$ (Giovannetti \& Mosse, 1980), após diafanização das raízes em $\mathrm{KOH} 10 \%$ e $\mathrm{H}_{2} \mathrm{O}_{2} 10 \%$ e coloração com fucsina ácida 0,01\% em lactoglicerol (Kormanic \& McGraw, 1984).

Para identificação das espécies de FMA nativos e avaliação final da densidade de esporos, foram feitas extrações a partir de amostras de $50 \mathrm{~g}$ de solo, por peneiramento úmido (Gerdemann \& Nicolson, 1963) seguido de centrifugação em água e sacarose (Jenkins, 1964).

O incremento no crescimento obtido com a inoculação foi calculado segundo a fórmula de Edginton et al. (1971) adaptada, tendo por base o valor médio do tratamento específico com FMA e do controle sem inoculação.

Os dados foram submetidos à análise de variância e as médias comparadas pelo teste de Tukey a 5\% de probabilidade, utilizando-se o programa SANEST (Zonta et al., 1984). Para análise, foram transformados os dados do número de folhas em $(x+0,5)^{0,5}$, densidade de esporos em $\log (x+1)$ e colonização de raízes em arc sen. Foram considerados, tanto para os tratamentos quanto para regressão e correlação, os níveis de probabilidade de $1 \%$ e $5 \%$.

Na análise de regressão e nas estimativas de correlação, foi utilizado o programa NTIA da Embrapa (1996).

\section{Resultados e Discussão}

Na rizosfera do maracujazeiro-doce em plantio comercial, foram identificadas 16 espécies de FMA, que constituíram o inóculo de FMA nativos: Acaulospora excavata Ingleby \& Walker, A. foveata Trappe \& Janos, A. longula Spain \& Schenck, A. rehmii Sieverding \& Toro, A. scrobiculata Trappe, A. tuberculata Janos \& Trappe, Entrophospora colombiana Spain \& Schenck, E. kentinensis Wu \& Liu, Glomus aggregatum (Schenck \& Smith) Koske, G. coremioides (Berk. \& Br.) Morton \& Redecker, G. geosporum (Nicol. \& Gerd.) Walker, G. glomerulatum Sieverding, G. macrocarpum Tul. \& Tul., G. sinuosum (Gerd. \& Bakshi) Almeida \& Schenck, Scutellospora heterogama e S. weresubiae Koske \& Walker. Algumas dessas espécies, que representam quatro gêneros de Glomeromycota, foram referidas anteriormente na rizosfera de fruteiras na região Nordeste (Melo et al., 1997).

Os FMA induziram respostas diferenciadas, com relação ao tempo, para formação das mudas de maracujazeiro-doce. Os tratamentos com FMA nativos e
Gigaspora albida promoveram maior crescimento das mudas, em relação ao controle, a partir de 30 dias após a inoculação, enquanto benefícios com a inoculação de $S$. heterogama somente foram observados a partir de 45 dias, quando todos os tratamentos com inoculação diferiram do controle (Tabela 1).

Esses resultados parecem indicar que os fungos nativos estavam mais adaptados ao hospedeiro e, portanto, podem ter colonizado mais rapidamente, enquanto os introduzidos demoraram mais a colonizar a muda e demonstrar efetividade. Fato semelhante ocorreu em mudas de cacaueiro, que responderam melhor à inoculação de fungos nativos do que de introduzidos (Cuenca et al., 1990).

Ao contrário desses resultados, Martins et al. (2000) observaram que fungos nativos da rizosfera de mamoeiro não foram efetivos. Em relação a FMA introduzidos, Costa et al. (2001) constataram que a inoculação de Gigaspora margarita Becker \& Hall começou a beneficiar o crescimento de aceroleiras, 56 dias após a inoculação, enquanto no tratamento com Glomus etunicatum Becker \& Gerd., isso só ocorreu após 84 dias.

As mudas associadas a $S$. heterogama atingiram a altura ideal $(15 \mathrm{~cm})$ aos 65 dias após a inoculação, com presença de gavinhas e formação da sétima folha, critérios referidos como condição para o transplantio ao campo (Ruggiero et al., 1996) (Tabela 1). As mudas controle apresentaram-se atrofiadas e com coloração verde-escura, típica da deficiência em fósforo.

Tabela 1. Efeito da inoculação de FMA na altura, diâmetro do caule e número de folhas e incremento produzido em mudas de maracujazeiro-doce, em casa de vegetação(1).

\begin{tabular}{|c|c|c|c|c|}
\hline \multirow[t]{2}{*}{ Tratamento de inoculação } & \multicolumn{3}{|c|}{ Dias } & \multirow{2}{*}{$\begin{array}{c}\text { Incremento aos } \\
65 \text { dias }(\%)\end{array}$} \\
\hline & 30 & 45 & 65 & \\
\hline & \multicolumn{3}{|c|}{ Altura $(\mathrm{cm})$} & \\
\hline Scutellospora heterogama & $3,45 b$ & $5,68 \mathrm{a}$ & $14,50 \mathrm{a}$ & 132 \\
\hline Gigaspora albida & $3,96 a$ & $6,20 \mathrm{a}$ & $13,74 \mathrm{a}$ & 119 \\
\hline FMA nativos & $4,12 \mathrm{a}$ & $6,11 \mathrm{a}$ & $10,78 b$ & 72 \\
\hline Controle & $3,38 \mathrm{~b}$ & $4,06 \mathrm{~b}$ & $6,25 \mathrm{c}$ & \\
\hline \multirow[t]{2}{*}{$\mathrm{CV}(\%)$} & 14,63 & 16,84 & 38,92 & \\
\hline & \multicolumn{3}{|c|}{ Diâmetro do caule (mm) } & \\
\hline Scutellospora heterogama & $1,78 b$ & $2,29 a$ & $3,45 \mathrm{a}$ & 45 \\
\hline Gigaspora albida & $1,76 b$ & $2,34 \mathrm{a}$ & $3,30 \mathrm{a}$ & 39 \\
\hline FMA nativos & $1,92 \mathrm{a}$ & $2,39 a$ & $3,25 \mathrm{a}$ & 37 \\
\hline Controle & $1,79 \mathrm{~b}$ & $1,96 \mathrm{~b}$ & $2,37 \mathrm{~b}$ & \\
\hline \multirow[t]{2}{*}{$\mathrm{CV}(\%)$} & 9,45 & 10,55 & 13,21 & \\
\hline & \multicolumn{3}{|c|}{ Número de folhas } & \\
\hline Scutellospora heterogama & $4,51 b c$ & $7,11 \mathrm{a}$ & $9,92 \mathrm{a}$ & 59 \\
\hline Gigaspora albida & $4,68 \mathrm{ab}$ & $7,49 a$ & $9,92 \mathrm{a}$ & 59 \\
\hline FMA nativos & $5,12 \mathrm{a}$ & $7,77 \mathrm{a}$ & $10,23 a$ & 64 \\
\hline Controle & $4,16 \mathrm{c}$ & $5,32 b$ & $6,22 b$ & \\
\hline $\mathrm{CV}(\%)$ & 6,78 & 8,63 & 11,73 & \\
\hline
\end{tabular}

(1)Médias de dois tratamentos de desinfestação e quatro doses de P no solo; médias seguidas da mesma letra não diferem entre si pelo teste de Tukey a 5\% de probabilidade. 
Segundo Borges et al. (1995), o tempo mínimo requerido para o transplantio do maracujazeiro-doce, em condições de adubação fosfatada utilizando-se $1 \mathrm{~kg}$ de superfosfato simples por $\mathrm{m}^{3}$ de solo, foi alcançado em 104 dias. A redução no tempo de produção de mudas obtida neste trabalho, com aplicação de FMA, também foi observada em aceroleiras (Costa et al., 2001) e maracujazeiro-amarelo (Cavalcante et al., 2002).

Apesar da ausência de especificidade na simbiose micorrízica, os isolados de FMA podem ter preferência na colonização por determinado hospedeiro, sendo a eficiência simbiótica governada pelos genótipos da planta e do fungo, assim como pelas condições ambientais (Sieverding, 1991).

Nas mudas de maracujazeiro-doce, as melhores respostas em altura e número de folhas foram obtidas, inicialmente (30 dias), nos tratamentos com G. albida e com FMA nativos, enquanto o efeito da inoculação de S. heterogama foi somente observado a partir dos 45 dias (Tabela 1). Isto pode ocorrer com algumas espécies de FMA, que demoram para colonizar o hospedeiro e produzir micélio externo, retardando a absorção dos nutrientes (Smith \& Read, 1997).

No maracujazeiro-amarelo, S. heterogama também produziu efeito tardio - 70 dias após a inoculação (Cavalcante et al., 2002). Graça et al. (1991) mencionaram que a colonização por este FMA, em maracujazeiro-amarelo, ocorreu apenas quando as plantas estavam associadas a Azospirillum brasilense, bactéria fixadora de nitrogênio.

Maiores incrementos na altura (132\%) e diâmetro do caule (45\%) foram observados nas mudas associadas com S. heterogama, enquanto G. albida e FMA nativos proporcionaram incrementos de $119 \%$ e $72 \%$ na altura e de $39 \%$ e $37 \%$ no diâmetro do caule, respectivamente. O maior incremento em número de folhas (64\%) ocorreu no tratamento com fungos nativos (Tabela 1). Benefícios no desenvolvimento foliar ocorreram em todas as mudas com inoculação, que apresentaram incrementos acima de 130\% (Tabela 2). Esse

Tabela 2. Efeito da inoculação de FMA na área foliar e respectivo incremento produzido em mudas de maracujazeiro-doce ${ }^{(1)}$.

\begin{tabular}{lcc}
\hline Tratamento de inoculação & Área foliar $\left(\mathrm{cm}^{2}\right)$ & Incremento $(\%)$ \\
\hline Scutellospora heterogama & $242 \mathrm{a}$ & 178 \\
Gigaspora albida & $217 \mathrm{a}$ & 149 \\
FMA nativos & $207 \mathrm{a}$ & 137 \\
Controle & $87 \mathrm{~b}$ & \\
\hline
\end{tabular}

${ }^{\left.{ }^{1}\right)}$ Médias de dois tratamentos de desinfestação e quatro doses de $\mathrm{P}$ no solo; médias seguidas da mesma letra não diferem entre si a 5\% de probabilidade pelo teste de Tukey; o coeficiente de variação foi de $47,63 \%$. efeito ocorreu independentemente do nível de $\mathrm{P}$ e da condição do solo. Incrementos diferenciados no crescimento de mudas com FMA também foram observados em mamoeiro (Martins et al., 2000) e maracujazeiro (Cavalcante et al., 2002).

Interações significativas entre a inoculação de FMA e os tratamentos de desinfestação do solo foram observadas no diâmetro do caule (Tabela 3). Nos dois tratamentos de solo as mudas micorrizadas apresentaram caule com maior diâmetro, quando comparadas ao controle. No tratamento sem FMA, maior diâmetro do caule ocorreu em solo não desinfestado, como esperado, considerando-se que a desinfestação do solo elimina os propágulos de fungos micorrízicos e demais microrganismos.

Na interação entre FMA e doses de P, observou-se regressão quadrática, aos 45 e 65 dias, para a altura de mudas de maracujazeiro-doce e, aos 65 dias, para o diâmetro do caule (P x G. albida e P x S. heterogama). Isso indica que o crescimento das mudas, com inoculação desses fungos, foi dependente de $\mathrm{P}$, com pontos de máximo entre 15,24 e 16,81 $\mathrm{mg} \mathrm{dm}^{-3}$. Houve regressão linear para o tratamento sem inoculação em relação ao diâmetro do caule, evidenciando-se a necessidade de adubação fosfatada na ausência de FMA. Regressão linear também foi observada em relação ao número de folhas (30 dias), entre G. albida x P e S. heterogama $\mathrm{x} P$, indicando que a produção foliar, nesse período, também dependeu da adição de $\mathrm{P}$, quando as mudas estavam associadas a esses fungos (Tabela 4).

Após 65 dias da inoculação, foram observadas interações significativas na altura e biomassa seca da parte aérea das mudas, entre $\mathrm{P} x$ solo desinfestado $\mathrm{x}$ S. heterogama e G. albida e P x solo não desinfestado x S. heterogama e G. albida, apresentando regressão quadrática. Isso evidencia que, além da fertilidade, a presença de outros organismos, juntamente com os demais fatores do solo, influencia a resposta da planta à inoculação. Para estas condições de solo e FMA, há

Tabela 3. Efeito da inoculação de FMA e da desinfestação do solo no diâmetro do caule de mudas de maracujazeirodoce aos 65 dias, em casa de vegetação(1).

\begin{tabular}{lcc}
\hline Tratamento de inoculação & \multicolumn{2}{c}{ Diâmetro do caule (mm) } \\
\cline { 2 - 3 } & Solo desinfestado & Solo não desinfestado \\
\hline Scutellospora heterogama & $3,46 \mathrm{aA}$ & $3,45 \mathrm{aA}$ \\
Gigaspora albida & $3,33 \mathrm{aA}$ & $3,17 \mathrm{aA}$ \\
FMA nativos & $3,32 \mathrm{aA}$ & $3,29 \mathrm{aA}$ \\
Controle & $2,10 \mathrm{bB}$ & $2,65 \mathrm{bA}$ \\
\hline
\end{tabular}

(1)Médias de quatro doses de P no solo; médias seguidas da mesma letra, minúsculas na coluna e maiúsculas na linha, não diferem entre si a 5\% de probabilidade pelo teste de Tukey. 
um ponto máximo de $\mathrm{P}$ no qual o benefício da inoculação é mais evidente e, quando este nível é ultrapassado, as mudas não respondem mais à fertilização fosfatada. Assim, a dose ideal de P, para o máximo desenvolvimento das mudas de maracujazeiro-doce, ficou entre 14,85 e 16,69 $\mathrm{mg} \mathrm{dm}^{-3}$, a depender do fungo inoculado e da condição do solo.

Para biomassa seca da parte aérea, houve regressão linear entre $\mathrm{P}$ x controle $\mathrm{x}$ solo desinfestado, indicando a necessidade da adição de doses mais elevadas de $\mathrm{P}$ na ausência de FMA. Da mesma forma, para o diâmetro ajustou-se regressão quadrática entre $\mathrm{P} \times \mathrm{G}$. albida $\mathrm{X}$ solo desinfestado ou não desinfestado e regressão linear entre $\mathrm{P} \times \mathrm{S}$. heterogama x solo desinfestado; $\mathrm{P}$ x controle x solo desinfestado ou não (Tabela 4), con- firmando que existe uma faixa ótima de $\mathrm{P}$ ou ponto de máximo desenvolvimento no qual o fungo exerce alta eficiência simbiótica (Saggin-Júnior \& Siqueira, 1996).

Resultados semelhantes foram observados por Trindade et al. (2000) em mudas de mamoeiro, onde a aplicação de $\mathrm{P}$ ao solo também promoveu respostas quadráticas no crescimento das mudas, com a inoculação de fungos nativos, apresentando resposta crescente até doses de $80 \mathrm{mg} \mathrm{dm}^{-3}$. De modo semelhante, em Citrus limonia Osbeck, maior altura ocorreu nos tratamentos com dose intermediária de $\mathrm{P}\left(100 \mathrm{mg} \mathrm{dm}^{-3}\right)$, enquanto na mais alta dose desse nutriente no solo (200 $\mathrm{mg} \mathrm{dm}^{-3}$ ) as mudas micorrizadas não se desenvolveram (Antunes \& Cardoso, 1991).

Tabela 4. Equação de regressão ajustada entre doses de $\mathrm{P}\left(\mathrm{mg} \mathrm{dm}^{-3}\right)$ e FMA, para altura (45 e 65 dias), diâmetro do caule (65 dias) e número de folhas (30 dias), e entre doses de $\mathrm{P}\left(\mathrm{mg} \mathrm{dm}^{-3}\right)$, FMA e desinfestação do solo, para altura (65 dias), diâmetro do caule (30 dias) e biomassa seca da parte aérea (65 dias), de mudas de maracujazeiro-doce.

\begin{tabular}{|c|c|c|c|}
\hline Tratamento de inoculação & Equação & $\mathrm{R}^{2}$ & Ponto de máximo \\
\hline $\begin{array}{l}\text { Scutellospora heterogama } \\
\text { Gigaspora albida }\end{array}$ & $\begin{array}{c}\text { Altura aos 45 dias } \\
Y=-0,785001+0,8540626 x-0,02539063 x^{2} \\
Y=-3,943125+1,3545313 x-0,04082031 x^{2}\end{array}$ & $\begin{array}{l}0,95^{*} \\
0,99^{* *}\end{array}$ & $\begin{array}{l}16,81 \\
16,59\end{array}$ \\
\hline $\begin{array}{l}\text { Scutellospora heterogama } \\
\text { Gigaspora albida }\end{array}$ & $\begin{array}{c}\text { Altura aos 65 dias } \\
Y=-33,624380+6,9607821 x-0,22832035 x^{2} \\
Y=-27,630624+5,8935935 x-0,19042968 x^{2}\end{array}$ & $\begin{array}{l}0,99^{* *} \\
0,99^{* *}\end{array}$ & $\begin{array}{l}15,24 \\
15,47\end{array}$ \\
\hline $\begin{array}{l}\text { Scutellospora heterogama } \\
\text { Gigaspora albida } \\
\text { Controle }\end{array}$ & $\begin{array}{c}\text { Diâmetro do caule aos } 65 \text { dias } \\
Y=-0,526876+0,5348438 x-0,01621094 x^{2} \\
Y=-0,476875+0,5285938 x-0,01699219 x^{2} \\
Y=-1,385000+0,0709375 x\end{array}$ & $\begin{array}{l}0,99^{* *} \\
0,97^{* *} \\
0,81^{* *}\end{array}$ & $\begin{array}{l}16,49 \\
15,55 \\
-\end{array}$ \\
\hline $\begin{array}{l}\text { Scutellospora heterogama } \\
\text { Gigaspora albida }\end{array}$ & $\begin{array}{l}\text { Número de folhas aos } 30 \text { dias } \\
Y=1,967147+0,0193893 x \\
Y=1,937131+0,0243133 x\end{array}$ & $\begin{array}{l}0,84^{* *} \\
0,87^{*}\end{array}$ & - \\
\hline $\begin{array}{l}\text { Scutellospora heterogama } \\
\text { Gigaspora albida }\end{array}$ & $\begin{array}{c}\text { Altura aos } 65 \text { dias (solo desinfestado) } \\
Y=-34,553749+6,7259372 x-0,21835937 x^{2} \\
Y=-25,217500+5,1981250 x-0,16171875 x^{2}\end{array}$ & $0,86^{* *}$ & $\begin{array}{l}15,40 \\
16,07\end{array}$ \\
\hline $\begin{array}{l}\text { Scutellospora heterogama } \\
\text { Gigaspora albida }\end{array}$ & $\begin{array}{c}\text { Altura aos } 65 \text { dias (solo não desinfestado) } \\
\mathrm{Y}=-17,435001+4,3868753 \mathrm{x}-0,14765626 \mathrm{x}^{2} \\
\mathrm{Y}=-14,421249+3,5959373 \mathrm{x}-0,11523437 \mathrm{x}^{2}\end{array}$ & $\begin{array}{l}0,73^{* *} \\
0,82^{*}\end{array}$ & $\begin{array}{l}14,85 \\
15,60\end{array}$ \\
\hline $\begin{array}{l}\text { Scutellospora heterogama } \\
\text { Gigaspora albida } \\
\text { Controle }\end{array}$ & $\begin{array}{l}\text { Diâmetro do caule aos } 30 \text { dias (solo desinfestado) } \\
\mathrm{Y}=1,215000+0,0431250 \mathrm{x} \\
\mathrm{Y}=2,951250-0,1971875 \mathrm{x}+0,00742187 \mathrm{x}^{2} \\
\mathrm{Y}=1,470000+0,0200000 \mathrm{x}\end{array}$ & $\begin{array}{l}0,86^{* *} \\
0,99^{* *} \\
0,91^{*}\end{array}$ & $\begin{array}{c}- \\
13,28 \\
-\end{array}$ \\
\hline $\begin{array}{l}\text { Gigaspora albida } \\
\text { Controle }\end{array}$ & $\begin{array}{c}\text { Diâmetro do caule aos } 30 \text { dias (solo não desinfestado) } \\
\mathrm{Y}=0,630000+0,1706250 \mathrm{x}-0,00546875 \mathrm{x}^{2} \\
\mathrm{Y}=1,475000+0,0187500 \mathrm{x}\end{array}$ & $\begin{array}{l}0,75^{*} \\
0,76^{*}\end{array}$ & $\begin{array}{c}15,6 \\
-\end{array}$ \\
\hline $\begin{array}{l}\text { Scutellospora heterogama } \\
\text { Gigaspora albida } \\
\text { Controle }\end{array}$ & $\begin{array}{c}\text { Biomassa seca da parte aérea aos } 65 \text { dias } \\
\text { (solo desinfestado) } \\
\mathrm{Y}=-4,048500+0,808065 \mathrm{x}-0,02507813 \mathrm{x}^{2} \\
\mathrm{Y}=-2,382125+0,5308437 \mathrm{x}-0,01589844 \mathrm{x}^{2} \\
\mathrm{Y}=-0,312750+0,0497500 \mathrm{x}\end{array}$ & $\begin{array}{l}1,00^{* *} \\
0,86^{* *} \\
0,77^{*}\end{array}$ & $\begin{array}{c}16,11 \\
16,69 \\
-\end{array}$ \\
\hline $\begin{array}{l}\text { Scutellospora heterogama } \\
\text { Gigaspora albida }\end{array}$ & $\begin{array}{l}\text { Biomassa seca da parte aérea aos } 65 \text { dias } \\
\text { (solo não desinfestado) } \\
Y=-0,984000+0,3553750 \mathrm{x}-0,01093750 \mathrm{x}^{2} \\
\mathrm{Y}=-1,629250+0,0495000 \mathrm{x}-0,01250000 \mathrm{x}^{2}\end{array}$ & $\begin{array}{l}0,87^{* *} \\
0,99^{* *}\end{array}$ & $\begin{array}{l}16,24 \\
16,38\end{array}$ \\
\hline
\end{tabular}

${ }^{*}$ e ${ }^{* *}$ Significativo a $5 \%$ e a $1 \%$ de probabilidade, respectivamente, pelo teste de Tukey a $5 \%$ de probabilidade. 
Segundo Nogueira \& Cardoso (2000), o aumento de $\mathrm{P}$ pode ter influência negativa sobre o micélio externo ativo do fungo micorrízico. Abbott \& Robson (1986) apresentaram várias hipóteses sobre a depressão no desenvolvimento de mudas, entre as quais: competição entre planta e fungo micorrízico por $\mathrm{P}$, toxicidade do $\mathrm{Pe}$ competição por fotossintatos entre endófito e planta hospedeira.

Os tratamentos com FMA introduzidos apresentaram maior produção de esporos e colonização micorrízica do que aqueles com FMA nativos, nas duas condições de solo. G. albida produziu mais esporos em solo desinfestado, enquanto os FMA nativos se multiplicaram melhor em solo não desinfestado (Tabela 5). Cavalcante et al. (2002) também encontraram esporulação de G. albida reduzida em solo não desinfestado e cultivado com maracujazeiro-amarelo. Embora tenham sido observados alguns esporos de FMA no solo desinfestado (controle), estes não foram capazes de colonizar as raízes (Tabela 5).

G. albida produziu maior colonização de raízes do que $S$. heterogama, porém isso não refletiu em maior eficiência desse FMA em promover o crescimento das mudas (Tabela 5). Em cacaueiro, Cuenca et al. (1990) também observaram que a eficiência dos FMA foi independente do grau de colonização produzido nas plantas.

Houve correlação entre os dados de colonização de raízes, densidade de esporos e características de crescimento das mudas de maracujazeiro-doce, independentemente da inoculação micorrízica e dos níveis de $\mathrm{P}$ utilizados. As correlações foram consideradas fortes $(\mathrm{r}=0,63 ; \mathrm{P}<0,74)$ entre colonização de raízes $\mathrm{x}$ altura da planta, diâmetro do caule, número de folhas e área foliar e entre colonização de raízes x densidade de esporos. Correlações médias $(r=0,37 ; \mathrm{P}<0,49)$ ocorreram entre densidade de esporos $\mathrm{x}$ altura da planta, diâmetro do caule e área foliar. Não houve correlação entre densidade de esporos e número de folhas (Tabela 6). Costa et al. (2001) também observaram correla-

Tabela 5. Densidade de esporos na rizosfera e colonização micorrízica em mudas de maracujazeiro-doce, aos 65 dias após a inoculação ${ }^{(1)}$.

\begin{tabular}{lrrrrrr}
\hline Tratamento de inoculação & \multicolumn{2}{c}{$\begin{array}{c}\text { Número de esporos } \\
\text { por 50 g de solo }\end{array}$} & & \multicolumn{2}{c}{$\begin{array}{c}\text { Colonização } \\
\text { micorrízica (\%) }\end{array}$} \\
\cline { 2 - 3 } \cline { 5 - 6 } & \multicolumn{2}{c}{$\mathrm{SD}^{(2)}$} & $\mathrm{SND}^{(3)}$ & & $\mathrm{SD}^{(2)}$ & $\mathrm{SND}^{(3)}$ \\
\hline Scutellospora heterogama & $238 \mathrm{aA}$ & $189 \mathrm{aA}$ & & $73 \mathrm{bA}$ & $77 \mathrm{aA}$ \\
Gigaspora albida & $257 \mathrm{aA}$ & $187 \mathrm{aB}$ & & $85 \mathrm{aA}$ & $72 \mathrm{aA}$ \\
FMA nativos & $6 \mathrm{bB}$ & $13 \mathrm{bA}$ & & $54 \mathrm{cA}$ & $52 \mathrm{bA}$ \\
Controle & $4 \mathrm{bA}$ & $4 \mathrm{cA}$ & & $0 \mathrm{~dB}$ & $18 \mathrm{cA}$ \\
\hline
\end{tabular}

(1)Médias seguidas da mesma letra, minúsculas na coluna e maiúsculas na linha, não diferem entre si a 5\% de probabilidade pelo teste de Tukey; o coeficiente de variação, em relação à densidade de esporos e à colonização micorrízica, foi de $14,66 \%$ e $21,00 \%$, respectivamente. (2)Solo desinfestado. ${ }^{(3)}$ Solo não desinfestado.
Tabela 6. Correlação entre parâmetros de crescimento de mudas de maracujazeiro-doce e de FMA, 65 dias após a inoculação, em casa de vegetação.

\begin{tabular}{cccc}
\hline Parâmetro $^{(1)}$ & Coeficiente (R) $^{* *}$ & Parâmetro & Coeficiente (R) \\
\hline CR x AP & $0,6326^{* *}$ & DE x AP & $0,4921^{* *}$ \\
CR x DC & $0,6801^{* *}$ & DE x DC & $0,3728^{*}$ \\
CR x NF & $0,6963^{* *}$ & DE x NF & $0,3264^{\text {ns }}$ \\
CR x AF & $0,7091^{* *}$ & DE x AF & $0,4422^{*}$ \\
CR x DE & $0,7412^{* *}$ & & \\
\hline
\end{tabular}

${ }^{(1)} \mathrm{CR}$ : colonização de raízes; DE: número de esporos $50 \mathrm{~g}^{-1}$ de solo; AP: altura da planta; DC: diâmetro do caule; NF: número de folhas; AF: área foliar. ${ }^{*} \mathrm{e}^{* *}$ Significativo a $5 \%$ e a $1 \%$ de probabilidade, res-

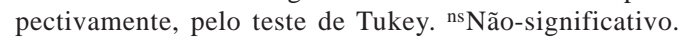

ções, de médias a fortíssimas, entre parâmetros de crescimento das plantas; entretanto, ao contrário do observado neste trabalho, não houve correlação entre esses parâmetros e a porcentagem de colonização.

\section{Conclusões}

1. O benefício da micorrização em maracujazeirodoce começa a partir de 30 dias após a inoculação e depende do isolado de FMA associado, podendo reduzir o tempo para produção de mudas.

2. O maracujazeiro-doce é beneficiado pela aplicação de FMA em solo desinfestado ou não, com até $16 \mathrm{mg} \mathrm{dm}^{-3}$ de $\mathrm{P}$, enquanto a adubação acima desse nível inibe a atuação dos FMA nessa fruteira.

3. A eficiência simbiótica dos FMA no maracujazeiro-doce depende do grau de colonização das raízes.

\section{Agradecimentos}

A Bruno Tomio Goto, pela ajuda na identificação dos FMA; à Capes, pela bolsa concedida a Érika Cristina Teixeira dos Anjos; ao CNPq, pela bolsa fornecida a Leonor Costa Maia.

\section{Referências}

ABBOTT, L.K.; ROBSON, A.D. The effect of VA mycorrhizae on plant growth. In: POWELL, C.L.; BAGYARAJ, D.J. (Ed.). VA Mycorrhiza. 2.ed. Boca Raton: CRC Press, 1986, p.113-130.

ANTUNES, V.; CARDOSO, E.J.B.N. Growth and nutrient status of citrus plants as influenced by mycorrhiza and phosphorus application. Plant and Soil, v.11, p.11-19, 1991.

BORGES, A.L.; LIMA, A.A.; CALDAS, R.C. Adubação orgânica e química na formação de mudas de maracujazeiros. Revista Brasileira de Fruticultura, v.17, p.17-22, 1995.

CAVALCANTE, U.M.T.; MAIA, L.C.; COSTA, C.M.C.; CAVALCANTE, A.T.; SANTOS, V.F. Efeito de fungos micorrízicos arbusculares, da adubação fosfatada e da esterilização do solo no 
crescimento de mudas de maracujazeiro amarelo. Revista Brasileira de Ciência do Solo, v.26, p.1099-1106, 2002.

COSTA, C.M.C.; MAIA, L.C.; CAVALCANTE, U.M.T.; NOGUEIRA, J.M.C. Influência de fungos micorrízicos arbusculares sobre o crescimento de dois genótipos de aceroleira (Malpighia emarginata D.C.). Pesquisa Agropecuária Brasileira, v.36, p.893901, 2001.

CUENCA, G.; HERRERA, R.; MENESES, E. Effects of VA mycorrhiza on the growth of cacao seedlings under nursery conditions in Venezuela. Plant and Soil, v.126, p.71-78, 1990.

EDGINTON, L.V.; KHEW, K.L.; BARRON, G.L. Fungitoxic spectrum of benzinidazol compounds. Phytopathology, v.61, p.4244, 1971.

EMBRAPA. Centro Nacional de Pesquisa Tecnológica em Informática para a Agricultura. SWNTIA: programa NTIA, versão 4.2.1. Campinas, 1996.

GERDEMANN, J.W.; NICOLSON, T.H. Spores of mycorrhizal Endogone extracted from soil by wet sieving and decanting. Transactions of the British Mycological Society, v.46, p.235244, 1963.

GIOVANNETTI, M.; MOSSE, B. An evaluation of techniques for measuring vesicular arbuscular mycorrhizal infection in roots. New Phytologist, v.84, p.489-500, 1980.

GRAÇA, J.; MACHADO, J.O.; RUGGIERO, C.; ANDRIOLI, J.L. Eficiência de fungos endomicorrízicos e da bactéria Azospirillum brasilense sobre o desenvolvimento de mudas do maracujá (Passiflora edulis f. flavicarpa). Revista Brasileira de Fruticultura, v.13, p.125-130, 1991.

INTERNATIONAL CULTURE COLLECTION OF VESICULAR ARBUSCULAR MYCORRHIZAL FUNGI. Disponível em http:/ /www.invam.wvu.edu. Acesso em:> 2003.

JARSTFER, A.G.; SYLVIA, D.M. Inoculum production and inoculation strategies for vesicular-arbuscular mycorrhizal fungi. In: METTING, F.B. (Ed.). Soil microbial technologies: applications in agriculture, forestry and environmental management. New York: Marcel Dekker, 1992. p.349-377.

JENKINS, W.R.A. A rapid centrifugal-flotation technique for separating nematodes from soil. Plant Disease Reporter, v.48, p.692, 1964.

JORGE, L.A.C.; CAMPOS NETO, D.; LEONI, E.; CRESTANA, S. Aquisição de imagens de folhas. In: JORGE, L.A.C. Recomendações práticas para aquisição de imagens digitais analisadas através do SIARCS. São Carlos: Embrapa-CNPDIA, 1996. (Circular Técnica, 1).

KORMANIC, P.P.; MCGRAW, A.C. Quantification of vesiculararbuscular mycorrhizae in plant roots. In: SCHENCK, N.C. Methods and principles of mycorrhizal research. Minnesota: The American Phytopathological Society, 1984. p.37-45.

MARTINS, M.A.; GONÇALVES, G.F.; SOARES, A.C.F. Efeito de fungos micorrízicos arbusculares associados a compostos fenólicos, no crescimento de mudas de mamoeiro. Pesquisa Agropecuária Brasileira, v.35, p.1465-1471, 2000.
MELO, A.M.Y.; MAIA, L.C.; MORGADO, L.B. Fungos micorrízicos arbusculares em bananeiras cultivadas no Vale do Submédio São Francisco. Acta Botânica Brasílica, v.11, p.115121, 1997.

NOGUEIRA, M.A.; CARDOSO, E.J.B.N. Produção de micélio externo por fungos micorrízicos arbusculares e crescimento da soja em função de doses de fósforo. Revista Brasileira de Ciência do Solo, v.24, p.329-338, 2000.

NOVAIS, R.F.; SMYTH, T.J. Fósforo em solo e planta em condições tropicais. Viçosa: UFV, 1999. 399p.

RUGGIERO, C.; SÃO JOSÉ, A.R.; VOLPE, C.A.; OLIVEIRA, J.C.; DURIGAN, J.F.; BAUMGARTNER, J.G.; SILVA, J.R.; NAKAMURA, K.; FERREIRA, M.E.; KAVATI, R.; PEREIRA, V.P. Maracujá para exportação: aspectos técnicos da produção. Brasília: Ministério da Agricultura e do Abastecimento, Secretaria de Desenvolvimento Rural; Embrapa-SPI, 1996. 64p. (Publicações Técnicas Frupex, 19).

SAGGIN JÚNIOR, O.J.; SIQUEIRA, J.O. Micorrizas arbusculares em cafeeiro. In: SIQUEIRA, J.O. (Ed.). Avanços em fundamentos e aplicação de micorrizas. Lavras: Universidade Federal de Lavras, 1996. p.203-254.

SCHENCK, N.C.; PEREZ, Y. Manual for the identification of VA mycorrhizal fungi. $3^{\text {rd }}$ ed. Synergistic Publications, Gainesville, 1990.

SIEVERDING, E. Vesicular-arbuscular mycorrhiza management in tropical agrosystems. Eschborn: ZTZ, 1991. 371p.

SILVEIRA, S.V.; SOUZA, P.V.D.; KOLLER, O.C. Influência de fungos micorrízicos arbusculares sobre o desenvolvimento vegetativo de porta-enxertos de abacateiros. Pesquisa Agropecuária Brasileira, v.37, p.303-309, 2002.

SMITH, S.E.; READ, D.J. Mycorrhizal simbiosis. Califórnia: Academic Press, 1997. 506p.

TRINDADE, A.V.; SIQUEIRA, J.O.; ALMEIDA, E.P. Eficiência simbiótica de fungos micorrízicos arbusculares em solo não fumigado, para mamoeiro. Revista Brasileira de Ciência do Solo, v.24, p.505$513,2000$.

VASCONCELLOS, M.A.S.; BRANDÃO FILHO, J.U.T.; VIEITES, R.L. Maracujá-doce. In: BRUCKNER, C.H.; PICANÇO, M.C. (Ed.). Maracujá: tecnologia de produção, pós-colheita, agro-indústria, mercado. Porto Alegre: Cinco Continentes, 2001. p.33-49.

ZONTA, E.P.; MACHADO, A.A.; SILVEIRA JUNIOR, P. Sistema de análise estatística para microcomputadores (SANEST). Pelotas: UFPEL, Departamento de Matemática e Estatística, 1984.

YANO-MELO, A.M.; SAGGIN-JÚNIOR, O.J.; LIMA-FILHO, J.M.; MELO, N.F.; MAIA, L.C. Effect of arbuscular mycorrhizal fungi on the acclimatization of micropropagated banana plantlets. Mycorrhiza, v.9, p.119-123, 1999. 